\title{
L'écriture poétique de la Chine chez Shan Sa
}

\begin{abstract}
:
The Naked Zither is the last novel by Shan Sa written in French. Chinese origin and French by adoption, she always tries to tell about her country highlighting the poeticity of her text that distinguishes her from the other writers of her time. This article will be devoted to the analysis of one aspect of the author's writing techniques related to the Chinese culture that appears as ethnostylistique and that intends to be a vector for her poetic identity.
\end{abstract}

Key words: poetic - ethnostylistique ethnostylème- zither - China _ culture.

\section{Maroua DAROUI}

Faculté des Lettres et Langues

Université Mentouri

Constantine1

\section{Introduction :}

" Écrire en français, c'était pour moi la meilleure façon de faire le pont entre la Chine et la France. Parce que dans ces moments-là, les codes tombent. J'essaie de ne pas faire de roman exotique, de guide de la Chine. Écrire directement en français a cet avantage : on écrit un vrai roman. Les lecteurs voyagent dans un univers qui leur est totalement inconnu mais avec la facilité de la langue. Et j'espère que cette langue française est écrite de telle manière qu'à travers elle, on aperçoit ce qu'est la langue chinoise. C'est peut-être là ce qui fait le style de tous mes livres " ${ }^{(1)}$ 
Ces propos sont proférés par Shan Sa, auteure chinoise d'expression française ayant choisi le français comme langue d'expression littéraire, et faisant partie d'un ensemble d'auteurs qui s'accroit depuis le début du XXe siècle tels que Tcheng Ki-Tong (1851-1907) à Ling Xi (1972-), François Cheng (1929), ou encore Gao Xingjian (1940-).

Shan Sa cherche toujours, à travers ses romans historiques, à parler de la Chine tout en empruntant à la langue française ses formes narratives et en adaptant ses techniques d'écriture à la culture chinoise. Faisant partie des auteurs en situation d'exil, Shan Sa trouve en l'Hexagone un terrain qui lui permet d'écrire la Chine, d'en chanter les beautés et de laisser transparaître l'histoire singulière de son pays d'origine. Ainsi, le français se veut pour elle le moyen de démontrer et de mettre en exergue sa propre poétique ayant comme centre d'intérêt l'Histoire de la Chine.

Etant née et grandie en Chine, Shan Sa est issue d'un espace non francophone. Cependant, elle a décidé de quitter son pays d'origine, de rompre avec sa langue maternelle pour écrire dans une langue aux antipodes culturels et linguistiques de la sienne. Cette rupture avec la Chine est causée essentiellement par l'instabilité politique de la Chine contemporaine. En effet, les événements de Tiananmen constituent le point de tournant radical pour l'auteure qui se voit exilée en France pour fuir un malaise politique et social profond, néanmoins elle garde un lien d'encre et de papier avec son pays d'origine en le racontant, sur une terre étrangère, de manière sereine et calme, loin de tout type de conflit.

L'enracinement prôné par l'écrivaine se traduit dans ses textes de différentes manières et sur plusieurs plans. Nous envisageons, dans ce présent article, de mettre l'accent sur un aspect essentiel dans la poétique de Shan Sa, qui se traduit par la présence abondante d'éthnostylèmes qui se définissent comme étant des unités linguistiques ayant une charge sémantique liée à une culture précise. Ils sont alors selon Gervais Mendo $\mathrm{Ze}$ des « indices référentiels ou déictiques permettant de situer le texte par rapport à la culture, à la langue et à la société occurrente, celle des liens sources textuels $»^{(2)}$

Nous nous intéresserons, dans ce présent travail, à quelques éthnostylèmes récurrents qui se réfèrent à l'Histoire de la Chine et se distinguent par une importance singulière dans le roman.

Ainsi, la présente étude tire tout son intérêt du questionnement suivant : De quelle manière les éthnostylèmes convoqués par Shan Sa participent-ils à la création de sa poétique?

Nous tenterons, dans la suite de cet article, de répondre à ce questionnement. Notre démonstration s'appuiera sur la lecture éthnostylistique du roman La Cithare Nue de Shan Sa.

Ainsi, l'approche éthnostylistique nous semble être l'outil méthodologique le plus adéquat à notre recherche dans la mesure où une 
lecture éthnostylistique nous permettra de mettre en relief quelques éthnostylèmes chinois récurrents et significatifs adoptés par l'auteure avec à quoi ils se réfèrent, pour proposer, ensuite, une interprétation de la spécificité de la poétique qu'elle cherche à tisser dans son roman.

1. Shan Sa, l'aventure de raconter la Chine par une plume française.

Se positionnant entre deux pays, deux cultures, et en se servant de deux langues, Shan Sa se trouve au carrefour des langues chinoise et française. Ainsi, il nous semble nécessaire de mettre en lumière quelques éléments biographiques qui expliquent à quel point l'écriture poétique de l'auteure est caractérisée par un ancrage profond et l'indéfectible dans la Chine, autrement dit, dégager l'influence du contexte de production sur la langue d'écriture.

Née à Pékin en 1972, Shan Sa est une poétesse et romancière française d'origine chinoise. Son vrai nom est Yan $\mathrm{Ni}$, elle s'est appropriée un pseudonyme aussi poétique que son écriture et qui signifie, en chinois, le bruissement du vent entre les montagnes. Dotée d'un talent de poète, elle a publié dès son plus jeune âge en chinois des recueils de poésie : Les poèmes de Yan Ni(1983), La libellule rouge(1988), Neige(1989) et Que le printemps revienne(1990). Elle a également été élue membre de l'association des écrivains de Pékin en 1987 et a gagné le Concours National de poésie des enfants. La vie de Shan Sa se verra alors bouleversée en 1989, l'année des événements de Tien An Men ; elle quitte la Chine pour rejoindre son père qui enseigne à la Sorbonne en France. Shan Sa entame alors des études en philosophie, puis devient la secrétaire du Balthus. L'influence de ce dernier et de sa femme Setsuko, qui l'initiera aux cultures japonaise et française, sera prépondérante pour Shan Sa. Elle commence alors à publier en français : Porte de la paix céleste (1997) fut sa première publication qui reçoit le prix Goncourt du premier roman en 1997. Deux ans plus tard, son roman Quatre vies du saule a remporté le prix Casez, en 2001, elle est de nouveau récompensée par le prix Goncourt des lycées pour son roman La joueuse de go. Depuis lors, elle a publié les romans Les conspirateurs (2005), Impératrice (2003) et Alexandre et Alestira (2006).

2. De la stylistique à l'éthnostylistique, une nouvelle perspective émerge.

La stylistique, comme étant une discipline à part entière, prend en charge l'étude du fonctionnement du langage littéraire comme le confirme Molinié :

"L'objet majeur et éminent de la stylistique est le discours littéraire, la littérature. Plus exactement c'est le caractère spécifique de littérarité du discours, de la praxis langagière telle qu'elle est concrètement développée, réalisée à travers un régime bien particulier de fonctionnement du langage, la littérature " ${ }^{(3)}$ 
Elle s'avère insuffisante, car elle néglige complètement les conditions extralinguistiques dans lesquelles le discours a été émis et reçu. Ainsi, il nous semble nécessaire de s'intéresser aux notions de forme et de contenu comme complémentaires pour pouvoir identifier ce que l'éthnostylistique a apporté de plus au domaine des sciences du langage. En effet, le texte littéraire est selon MendoZe «une forme certes, mais aussi au contenu idéologico-culturel, et toute interprétation critique ne peut intégrer cette perspective référentielle sans reconsidérer la problématique saussurienne du signe linguistique ${ }^{(4)}$

Saussure, quant à lui, estime que le sens d'un texte ne peut être interprété que par sa forme car:

«La langue, martèle Saussure, est une forme et non une substance. Il n'y a rien de substantiel dans le langage: ce sont les différences, les oppositions qui donnent existence aux données du langage : ce qui distingue un signe, voilà tout ce qui le constitue. C'est la différence qui fait le caractère, comme elle fait la valeur et l'unité $\rangle^{(5)}$

De ce fait, étant un phénomène clos, le texte doit être décomposé pour pouvoir dégager sa cohésion interne. Rajoutons à cela, qu'à côté du structuralisme, d'autres théories ont émergé dans le domaine des sciences du langage, et qui prônent l'idée qu'un texte coupé de son cadre énonciatif ne peut véhiculer qu'imparfaitement le sens.

En effet, chaque production littéraire comporte des marques qui démontrent l'acte individuel de l'utilisation de la langue et s'inscrit toujours dans une dynamique socioculturelle environnante qui détermine le style d'un auteur. De ce point de vue, nous pouvons dire qu'il n'existe pas une stylistique mais des stylistiques appropriées à chaque auteur. Dans ce sens, la stylistique structurale ne s'intéresse qu'à la forme du texte, celle de Bally étudie l'impact de la sensibilité sur les faits d'expression, la génétique de Spitzer étudie l'effet spirituel de l'auteur sur son texte et la sémiostylistique de Molinié analyse des caractérisèmes de la littérarité textuelle, quant à la toute nouvelle ethnostylistique de Gervais MendoZe, elle cherche dans le discours les modes particuliers d'expression des valeurs culturelles ainsi que l'étude des textes qui sont en relation étroite avec leur contexte d'énonciation.

\section{3 .Qu'est- ce- que l'éthnostylistique ?}

L'étude de la langue en tant qu'expression partielle ou totale de la société est l'objet majeur de l'ethnostylistique. En effet, Il n'y a pas de société sans langue ni de langue sans société qui la forge dans la pratique humaine et sociale, dans son rapport avec son environnement. Il y a une implication réciproque entre la société et la langue dans la mesure où l'une complète l'autre :

"Immédiatement la société est donnée avec le langage. La société à son tour ne tient ensemble que par l'usage commun de signes de communication. Immédiatement, le langage est donné avec la société. Ainsi chacune de ces 
deux entités, langage et société, implique l'autre. Il semblerait que l'on puisse et même qu'on doive les étudier ensemble, les découvrir ensemble, puisque ensemble elles sont nées " ${ }^{(6)}$

En fait, la langue conceptualise et interprète la société en transformant l'expérience humaine en signes linguistiques. L'étude de la langue en tant qu'expression d'une culture est en relation exclusive avec la situation de communication qui est l'objet essentiel de l'éthnostylistique ; cette nouvelle approche dans le domaine des sciences du langage concerne la relation qui lie la linguistique au monde extérieur, c'est - à - dire le contexte extralinguistique, les conditions de production et d'interprétation, qui contribuent essentiellement à la signification du discours, et prône l'idée selon laquelle le discours doit être envisagé dans un contexte ou dans un environnement contextuel précis.

L'éthnostylistique est un mot-valise, formé à partir de « ethnologie » et de «stylistique », cela signifie que cette approche développée par le professeur Gervais MendoZe en 2004 dans le cadre de la revue Langues et communication de l'université de Yaoundé, englobe ces deux disciplines, autrement dit, l'éthnostylistique est essentiellement une stylistique particulière qui se situe au carrefour de l'ethnologie et de la stylistique et qui s'intéresse à l'analyse du discours littéraire, en mettant en exergue aussi l'analyse des dimensions culturelle et sociale véhiculées par le texte littéraire.

A ce propos MendoZe affirme que :

"L'éthnostylistique est une stylistique qui a pour objet la critique du style des textes littéraires, pour procéder aux techniques d'analyse en sciences du langage, et pour finalité la prise en compte des conditions de production et de réception des textes ainsi que l'étude des modes particuliers d'expression des valeurs culturelles ${ }^{(7)}$

Il ajoute, un peu plus loin :

"L'éthnostylistique est à considérer, en outre, comme l'étude formelle du message linguistique en liaison avec l'ensemble des circonstances de communication et des conditions particulières de l'énonciation ${ }^{(8)}$

Chaque texte littéraire est l'expression d'une culture. En effet, l'auteur, par le biais de son texte, véhicule et fait connaître au monde sa propre vision des choses, ses pratiques sociales par sa langue, sa culture, son histoire. Shan Sa concrétise cet aspect avec excellence, car elle publie toujours des romans historiques parlant de l'histoire de la Chine à toutes ses époques.

Dans ce sens, l'éthnostylistique semble être l'approche la mieux adaptée à analyse que nous envisageons d'entreprendre, du moment qu'elle prône l'idée qu'il est obligatoire d'intégrer, à côté des pratiques proposées par les stylistiques (structurale, génétique, fonctionnelle...), la composante extra linguistique pour l'analyse d'un texte littéraire, car selon Fosso «le contexte extralinguistique est alors l'environnement matériel pertinent pour l'encodage 
et le décodage ${ }^{(9)}$; et permet, ainsi, de mettre en valeur une caractéristique spécifique dans la poétique de Shan Sa.

Il convient, à notre sens, de faire appel aux différents postes d'analyse proposés par Gervais MendoZe pour mettre en lumière les pratiques culturelles présentes dans La Cithare Nue. Dès lors, cette démarche se déploie en deux temps :

«Dans un premier temps, elle étudie le contexte d'énonciation, repéré à partir d'indices référentiels ou déictiques. Ceux-ci constituent des éthnostylèmes permettant de situer le texte par rapport à la culture, à la langue et à la société occurrente, celle des lieux-sources textuels. Elle se préoccupe ainsi des conditions dans lesquelles l'acte d'énonciation a pris place.

-Dans un deuxième temps, elle procède à l'étude des modalités du style de l'énoncé ainsi que sa dominante tonale, examine la structuration du texte, ses formes d'expression particulières, les caractéristiques de son écriture aux niveaux lexical, morphosyntaxique, rhétorique, etc. " ${ }^{(10)}$

\section{Lecture éthnostylistique de La Cithare Nue}

Tout d'abord, nous examinerons le contexte d'énonciation "relativement à l'ancrage idéologico-culturel de ce discours $\|^{(10)}$ dans le but de démontrer les pratiques dont il est question en se référant à des lieux de source précis. En effet, le contexte d'énonciation crée une relation de coprésence et de dépendance entre l'énonciateur et son coénonciateur.

A ce propos MendoZe déclare :

"Ces deux instances sont en rapport d'échange discursif dans un espace déterminé et à un moment donné. Les coordonnées du réel ( Je/Tu, Ici) Maintenant) doivent être repérées, analysées et interprétées par rapport à la culture, à la langue et à la société $\rangle^{(11)}$

Ainsi l'interprétation du contexte d'énonciation doit s'effectuer par rapport à la culture au sein de laquelle il a émergé.

4.1 Les toponymes : le fleuve de Yingzin, lieu témoin.

L'incipit de La Cithare Nue nous plonge directement dans la Chine des premiers âges, à une époque où l'Occident était aux mains des barbares. En effet, l'auteure cherche à situer l'histoire de la Chine dans l'Histoire universelle pour permettre au lecteur francophone d'assimiler et de comprendre la grandeur et le prestige de son pays. A ce propos elle entame son récit en racontant :

" Les grandes invasions barbares commencèrent au IIIème siècle. En l'Occident, les Francs, les Alamans, les Huns, les Gothes, les Germains, les Vandales firent irruption et déstabilisèrent l'Empire romain. En 476, l'abdication de l'empreur Romulus Augustule marqua son effondrement $\|^{(11)}$

L'auteure expose un bref panorama des multiples changements historiques et politiques de l'Empire chinois à partir de 476. Ces derniers sont 
caractérisés par la succession rapide et brève de multiples dynasties semant la mort et la haine, ravageant les villes et les campagnes, et nous livre, aussi, des informations sur les différentes circonstances qui ont participé à la construction de l'architecture idéologique et politique de son pays. Elle déclare :

«Durant la même prériode, les tribus nomades Xianbei, Xongnu, Di, Jiang déferlèrent sur l'Empire chinois. Elles provoquèrent la chute de la dynastie de Han, puis celle de la dynastie Jing, et forcèrent les Chinois à quitter leur terre et à se réfugier au sud du fleuve Yangzi »(12)

La présence massive de référents liés à l'histoire de la Chine dans le contexte d'énonciation permet, non seulement, d'assurer un lien entre le lecteur et l'univers diègétique dans lequel le texte a émergé, mais aussi de créer un effet d'ancrage dans le réel dans la mesure où l'auteure parle d'une Chine véridique et non imagée. Cet ancrage s'explique par une présence abondante de toponymes qui sont considérés comme des références à des lieux géographiques réellement existants en Chine.

. Donc, par ce lieu, elle permet une meilleure transmission de l'histoire de son pays et par conséquent une identité spécifique dans sa poétique.

Les extraits suivants illustrent nos propos :

«Durant la même période, les tribus nomades Xianbei, Xongnu, Di, Jiang déferlèrent sur l'Empire chinois. Elles provoquèrent la chute de la dynastie Han, puis celle de la dynastie Jin, et forcèrent les Chinois à quitter leur terre et se réfugier au sud du fleuve Yangzi. Le pays fut alors divisé en deux parties. Au sud du fleuve, les tribus nomades se sinisèrent. En adoptant l'administration et l'écriture chinoises, elles fondèrent leurs royaumes. Au sud, les Chinois continuèrent à s'unir autour du Fils Du Ciel, un empereur considéré comme ayant un mandat divin ${ }^{(13)}$

"Quand la grande invasion des Barbares mit l'armée chinoise en déroute, le clan quitta la Plaine du Milieu, franchit le fleuve Yangzi et se réfugia sur sa rive sud. Une nouvelle dynastie y fut fondée, portant le nom de Jin de l'Est ${ }^{(14)}$

« La Cour impériale dépêcha ses généraux qui enrôlèrent des criminels et firent appel aux brigands pour réprimer les insurgés. Les deux forces s'affrontèrent le long de la rive du sud du Yangzi ${ }^{(15)}$

"Le soleil se lève sur la ville de Jing Ko et son panneau laqué noir "Porte du Fleuve ». »"(16)

Nous constatons que l'occurrence toponymique « le fleuve de Yingzi » permet l'ancrage du récit dans un lieu réel, témoin des différentes batailles, guerres et dynasties qui se sont succédées sur ce lieu en racontant une Chine lors de deux périodes de son histoire, en l'an 400, lors de la dynastie de Jin de l'Est, et en l'an 581, sous le règne de la dynastie de Chen. Shan Sa cherche alors à dépayser le lecteur francophone en le transportant dans une aire géoculturelle nouvelle. 


\subsection{Les anthroponymes : raconter l'Histoire par une histoire authentique.}

Les personnages constituent un pivot essentiel dans un roman. Le personnage, en tant que tel, construit et représenté par le narrateur, est l'expression d'une culture parce qu'il est doté d'une charge sémantique liée à l'aire culturelle dans dont il est issu.

Dans La Cithare Nue, les personnages, à travers leurs noms, sont fortement culturalisés. En effet, sur un plan anthroponymique, l'auteur fait appel à des personnages authentiques. Ils renvoient à des personnalités chinoises réellement existantes et, par conséquent, à la culture chinoise et fonctionnent comme des culturèmes, c'est-à-dire des unités textuelles permettant de situer le texte relativement à la Chine . Ainsi, ils permettent de connoter l'aire culturelle de notre corpus.

Les exemples suivants illustrent nos propos :

«La Cithare portait sept cordes de soie nouvellement montées. Lorsqu'elle les effleura, l'instrument résonna, grave et solitaire, comme si on soufflait dans une corne barbare. Elle appartenait à la grande poétesse Cai Yan qui avait vécu quelques deux cents ans auparavant sous la dynastie Han. Son père Cai Yong, célèbre poète, calligraphe, joueur de cithare, avait parmi ses élèves des hommes qui deviendraient des seigneurs de la guerre et des maîtres de l'Empire chinois " ${ }^{(17)}$

" La dame Cai Yan, la fille du grand poète Cai Yong, a été enlevée par les nomades. Pendant douze ans, elle a vécu dans le vent du nord, parmi les chevaux et les moutons, et elle a composé dix-huit suites pour la cithare pour exprimer son chagrin et sa nostalgie. Elle a été rachetée par le général Cao Cao aux Barbares contre un char rempli d'or et de perles...lorsque Cai Yan est retournée dans la Plaine du Milieu, elle a offert sa cithare à Cao Zhi, le second fils du général. Fameux poète comme son père Cao Cao, Cao Zhi a été assassiné par son frère Cao Pei, jaloux de son talent. Cao Pei a usurpé le trône, renversé la dynastie Han et fondé la dynastie Wei $\|^{(18)}$

" il y a huit mois, $j$ "'en ai vendu une de la période des Trois Royaumes, laquelle, d'après la légende, appartenait au général Zhou Yu du royaume de $W u »^{(19)}$

\section{Etude de l'éthnostylème « La cithare "}

Etant un roman historique, La cithare nue met en filigrane la dimension culturelle de la Chine. Cette dernière se manifeste par le biais des ethnostylèmes qui constituent un élément essentiel dans la poétique de l'auteure. Les ethnostylèmes sont définis par Gervais Mando Ze comme étant :

«Des signes linguistiques pris sous le double plan de la compréhension et de l'extension. Le premier plan intègre la valeur autonomique du signe linguistique dans un système immanent tandis que le second insiste sur son caractère extra référentiel $»^{(20)}$. 
Donc, nous estimons que « la cithare» est une référence authentique d'un pays et dont l'auteure use pour permettre l'ancrage du texte dans sa culture d'origine.

L'élément paratextuel, le titre du roman, illustre parfaitement la notion d'ethnostylème, dans la mesure où ce dernier renvoie à un élément qui entre dans le cadre de la culture chinoise et qui est la cithare. Ce dernier est présent de manière récurrente tout au long de la diégèse et constitue, à côté des protagonistes, un actant à part entière dans le roman.

La cithare est un instrument musical très ancien d'origine chinoise inventé, selon la légende, par le dieu Fu Xi durant la Chine médiévale. La cithare est composée de sept cordes de soie et chaque corde symbolise une classe sociale de la société chinoise médiévale. La cithare est une composante fondamentale de la culture chinoise qui se transmet de génération en génération et ne se pratique que par les élites et les aristocrates de la société chinoise. Ainsi, les secrets du fonctionnement de la cithare ne se transmettent qu'oralement et laissent des partitions orales codées par des chiffres et de calligraphie chinoise, dont personne sauf les initiés peuvent déchiffrer le secret de sa sonorité.

La cithare joue dans le roman un rôle prépondérant, car elle unit, dans une Chine déchirée par la guerre, deux personnes de deux époques différentes et de deux classes sociales séparées et leur permet de vivre un amour et une passion impossibles.

En effet, les sept cordes de la cithare symbolisent les différentes classes sociales, la jeune mère, qui ne porte pas de nom dans le roman, est l'héritière d'un clan illustre de la Plaine Du Milieu qui est issue d'une classe de nobles appelée Hautes Portes.

Shan sa raconte :

"Elle descend d'un clan illustre de la Plaine du Milieu. Coule dans ses veines le sang de tous les nobles appelés les Hautes Portes se fréquentent et se marient entre eux depuis la nuit du temps. Constituant la plus haute caste de la société chinoise, ils ne paient pas d'impôts à la Cour, n'ont pas de devoirs envers les empereurs et vivent en autarcie à la campagne $\|^{(21)}$

Elle voit sa vie s'écrouler dans l'atrocité de la guerre de la Chine médiévale. Elle est enlevée par un soldat chinois dominé par la guerre qui la retient prisonnière et l'oblige à vivre dans un monde étranger. Elle voit son destin couler devant ses yeux, devenue veuve elle se consacre à l'éducation de ses enfants puis finit ses jours dans monastère de la Grande Compassion.

Shan Sa relate :

«Dans son chariot recouvert d'une tenture noire, la Jeune Mère somnole. Elle a perdu la notion du temps et ne voit plus le changement des saisons. La route militaire est un corridor sombre et froid isole du reste du monde. La vie militaire est rythmée par le vagissement des cors annonçant 
l'avancée et l'arrêt, par le roulement des tambours ordonnant l'attaque et le retrait. La jeune mère ignore la joie. Elle ne connaît que la tristesse. Vulnérable et désarmée, elle est obligée de suivre les hommes qui l'ont enlevée $\|^{(22)}$

Shen Feng, quant à lui, est un luthier pauvre, apparait près de deux siècles après la mort de la jeune mère. Etant passionné de la cithare, il mène une vie difficile et s'oblige à quitter sa vie pour fuir la guerre. En s'isolant, il rencontre l'âme de la jeune femme noble et tombe amoureux d'elle et partagent ensemble une passion commune pour la musique sacrée de la cithare.

Ainsi, la cithare brise toutes les règles sociales qui partagent et séparent la société chinoise. En vibrant ses cordes, elle entremêle les relations sociales sur une harmonie et une sonorité musicale communes et par le biais d'une écriture purement poétique qui permet à l'auteure de nouer un lien intime entre le sens qu'elle veut communiquer et la manière dont ce dernier se transmet à travers les mots qu'elle choisi.

En effet, le choix des mots qu'elle fait confère à son écriture une sensibilité à raconter la Chine et qui la distingue des autres auteurs chinois francophones. Ainsi, la création verbale qui se manifeste dans son texte lui confère le caractère de littérarité considéré comme le fondement de la poétique.

"La musique est un don. La musique engendre les mots, les mots engendrent la pensée, la pensée crée l'amant et l'amante. Ils naissent l'un pour l'autre. Ils meurent unis par la même musique. Ils se perdent, puis se retrouvent; ils oublient puis se souviennent quand résonne l'harmonie. Le temps est la musique ${ }^{(23)}$

\section{Conclusion}

En conclusion, le questionnement que nous nous sommes posé au départ de notre analyse nous semble être confirmé car l'écriture poétique de Shan Sa est très évocatrice, car elle interprète la culture chinoise par une plume française. En ayant recours à une écriture qualifiée de poétique, l'auteure nous plonge dans une Chine médiévale riche en pratiques culturelles originales qui se partagent tout au long de l'histoire par les protagonistes. Ces pratiques se traduisent essentiellement à travers "la cithare », éthnostylème se veut un vecteur de la poéticité de l'auteure qui fait de cet instrument un pont entre une Chine bouleversée par la guerre et le lecteur francophone d'aujourd'hui.

\section{Références bibliographiques}

Corpus :

- Shan Sa, La Cithare Nue, Albin Michel, Paris, 2010 Ouvrages théoriques :

- Stephanie CAMPBELL, La joueuse de go : entre la calligraphie et le français, McMaser University, Voix plurielles 8.1, 2011 
- Emile Benveniste, Problèmes de linguistique générale, Paris, Gallimard, 1966.

- Fosso, L'option ethnostylistique fondements épistémologiques,Langue et communication, $n^{\circ} 4,2004$.

- Gervais MendoZe, S...comme stylistique: propositions pour l'ethnostylistique, Paris, Editions L'Harmattan, 2009.

- Gervais MendoZe, Cahier d'un retour au pays natal. Aimé Césaire. Approche ethnostylistique. Paris : L'Harmattan, 2010.

- Georges Molinié, Eléments de stylistique française, Presse Universitaire de France, Paris, 1986.

Note :

1- Stephanie CAMPBELL, La joueuse de go : entre la calligraphie et le français, McMaser University, Voix plurielles 8.1, 2011, Page 123.

2-Gervais Mendo Ze, Cahier d'un retour au pays natal. Aimé Césaire. Approche ethnostylistique. Paris, :L'Harmattan, 2010, Page 37

3-Georges Molinié, Eléments de stylistique française, Paris, Presse

Universitaire de France, 1986, p02

4-Gervais Mendo Ze ,S comme stylistique, Harmattan, Paris, 2009, p 240.

5-ibid, p. 169.

6-Emile Benveniste, Problèmes de linguistique générale, Paris, Gallimard, p91

7- Gervais MendoZe, op. cit ,p226

8- ibid, p. 226.

9-Fosso, L'option ethnostylistique fondements épistémologiques :Langue et communication, $n^{\circ} 04,2004, p 51$

10- Gervais MendoZe, op,cit,p 434

10-Gervais MendoZe. Op. cit, p. 261.

11 - ibid,p. 232.

11-Shan Sa, La Cithare Nue, éditions Albain Michel, paris, 2010, p 7.

12- Ibid, p7.

13-Shan Sa, op, cit, p 7

14- Ibid, p13

15-Ibid, p29

16- Ibid,p39

17- Shan Sa, op, cit, p 28

18 Ibid, p 46

19-Shan Sa, op, cit, p 43

20- Gervais Mendo Ze. op. cit, p. 313

21- Shan Sa, op, cit, p 13

22- Shan Sa, op, cit p11

23-Ibid, 325 